\title{
Fremilab
}

FERMILAB-FN-0788-AD May 2006

\section{Residual Activation of Thin Accelerator Components*}

\author{
N.V. Mokhov, E.I. Rakhno**, I.L. Rakhno \\ Fermilab, P.O. Box 500, Batavia, Illinois 60510 \\ ${ }^{* *}$ North Central College, 30 North Brainard St., Naperville, Illinois 60540
}

May 18, 2006

\begin{abstract}
A method to calculate residual activation of thin accelerator components is presented. A model for residual dose estimation for thick objects made of arbitrary composite materials for arbitrary irradiation and cooling times is employed in this study. A scaling procedure is described to apply the model to thin objects with linear dimensions less than a fraction of a nuclear interaction length. The scaling has been performed for various materials and corresponding factors have been determined for objects of certain shapes (slab, solid and hollow cylinder) which are important from practical standpoint and can serve as models for beam pipes, magnets and collimators. Both contact residual dose and dose attenuation in air outside the objects were considered. A comparison between calculations and measurements performed at the Fermi National Accelerator Laboratory using a $120 \mathrm{GeV}$ proton beam is presented.
\end{abstract}

\section{Introduction}

Induced activation of accelerator components is an important issue from practical standpoint. After an accelerator shutdown or during normal operation various beam line components like collimators, magnets, beam pipes, windows and so forth reveal high induced activation. Handling and maintenance of such components can be extremely difficult and, therefore, correct prediction of their residual activity is of primary importance when planning on various hands-on and maintenance procedures.

Nowadays the most complete and correct procedure to predict residual activation of an object is the following: (i) calculation of residual nuclei distribution with a Monte Carlo code for a given irradiation scenario; (ii) determination of a $\gamma$-ray source using the obtained distribution and nuclear data on the decay chains and nuclear transmutation from

*Work supported by the Universities Research Association, Inc., under contract DE-AC02-76CH03000 with the U. S. Department of Energy. 
the CINDER library [1] and ORIGEN [2] or DeTra [3] code, respectively; (iii) calculation of the subsequent $\gamma$-ray transport through the object and dose distribution around it by means of a Monte Carlo code. In practice, however, a more simplified approach is often used to predict residual activation. Namely, contact residual dose for an irradiated object is determined using calculated distributions of star density and particle flux as well as precalculated star-to-dose and flux-to-dose conversion factors, respectively [4]. This approach is suitable for thick objects with linear dimensions exceeding some fraction of a nuclear interaction length. For thin objects, however, this procedure gives rise to an overestimated residual dose. Direct measurements performed at CERN in the early 70s [5] revealed that measured residual dose for thin objects is lower than predicted one within a factor of three and the disagreement varies depending on material and size of the object. Such a tendency of overestimating the measured dose can be explained taking into account the procedure to determine the above-mentioned conversion factors. Namely, an equilibrium condition is assumed to be valid for an object under consideration. For thin objects, however, the outgoing radiation can differ significantly from the incoming one and the equilibrium condition does not hold.

In this paper we describe briefly the essentials of the method developed previously [6][8] to determine the star-to-dose and flux-to-dose conversion factors for thick objects. In this context thick means infinite because no geometry considerations are taken into account at this stage (see the following section). After that a scaling procedure is introduced for taking into account the deviations from the equilibrium for thin objects. The scaling factors are calculated for a number of materials and objects of certain shapes that can serve as models for collimators, magnets and beam pipes. A comparison to measured residual doses is presented as well.

\section{Model for contact residual dose for thick objects}

While most of the values predicted with modern Monte Carlo codes for high energy accelerator environments can be obtained with a rather high accuracy, residual dose rates remain less reliable. Uncertainty up to a factor of three can be considered as typical. This is because of the complicated nature of this phenomenon and its high sensitivity to the composition of irradiated materials. In principle, a multi-step approach based on a hadron transport code (e.g., MARS [9] or FLUKA [10]) coupled to a nuclide transmutation inventory code (CINDER [1] or DeTra [3]), would provide the most reliable solution of activation problems - provided the hadron code is able to deliver adequate residual nuclide yields from high-energy interactions. In practice, however, one often uses an approach based on socalled $\omega$-factors that converts the star density (a density of inelastic nuclear interactions above $50 \mathrm{MeV}$ ) to a contact residual dose rate for various combinations of irradiation $\left(T_{i}\right)$ and cooling $\left(T_{c}\right)$ times (typically $T_{i}=30$ days, $T_{c}=1$ day). The concept of $\omega$-factors was introduced more than three decades ago [4]. It is based on the assumption that a high-energy interaction of a projectile hadron with a target nucleus or in other words, a star, generates a number of radioactive nuclei so that for the average resulting radioactivation one can perform a simple parametrization that depends only on the target material. Namely, for a 
semi-infinite body the residual dose rate on its surface is assumed to be described by the following expression:

$$
\frac{d D}{d t}=\omega \frac{d^{2} S}{d V d t}
$$

where $d^{2} S / d V d t$ is the star density production rate which is assumed to be uniform over the volume of the body. As can be seen [11], this model is a rather crude approach to reality. A number of Monte Carlo calculations has been performed to determine an appropriate set of the $\omega$-factors using the expression (1). In particular, it has been shown [12] that when defining $\omega$-factors, a $20 \mathrm{MeV}$ star threshold should be used instead of the historical $50 \mathrm{MeV}$ because of a non-zero contribution from spallation reactions in the 20-50 MeV region. No correlation was used between induced activation and target atomic mass. In addition, residual activation reveals a dependence on projectile energy spectrum. It should be noted also that the $\omega$-factors describe the residual dose only due to emission of $\gamma$-quanta [8]. As long as we do not consider very thin objects the contribution from gamma decays is dominating when compared to that due to beta decays. In addition, the dose due to the $\beta$ particles is not negligible only at small depths - up to a few millimiters in tissue-because of the short ranges of the particles.

An elaborate set of the $\omega$-factors was described in Refs. [6, 7]. New modules have been developed (see Ref.[6]) for the MARS14 and MARS15 codes [9] to substantially improve the reliability of the $\omega$-factor based predictions of residual dose rates in arbitrary composite materials for arbitrary irradiation and cooling times. The algorithm distinguishes three major energy groups responsible for radionuclide production: (1) above $20 \mathrm{MeV}$, (2) 1 to $20 \mathrm{MeV}$, and (3) below $0.5 \mathrm{eV}$. The energy groups were chosen to consider separately the most important nuclear reactions responsible for induced radioactivation in the regions: high energy inelastic interactions (mostly spallation reactions), threshold reactions $(n, 2 n)$, $(n, p)$ etc, and $(n, \gamma)$ reactions, respectively. Neutrons in the energy region from $0.5 \mathrm{eV}$ to 1 $\mathrm{MeV}$ do not produce a significant number of radionuclides. Detailed FLUKA calculations were performed for cascades induced by energetic hadrons in cylindrical samples of 17 elements: C, O, Na, Mg, Al, Si, K, Ca, Cr, Fe, Ni, Cu, Nb, Ag, Ba, W, Pb. Creation of the residual nuclides close to the cascade core was simulated. The decay chains of the created radionuclides were followed with the DeTra code in order to determine the emission rates of de-excitation photons for 12 hours $<T_{i}<20$ years and $1 \mathrm{sec}<T_{c}<20$ years. Corresponding dose rates on the outer surfaces were calculated from photon fluxes and related to the star density above $20 \mathrm{MeV}$ (first group), and neutron fluxes in two other energy groups. Results were collected in the database. This method essentially applies the optimum way of activation prediction described above to derive a set of material and time dependent $\omega-$ factors which are easy to use in a routine cascade simulation and should provide far better accuracy than the old approach.

A sophisticated interpolation algorithm, linked to this database, was created and implemented into the MARS code. As an example, numerical values of the $\omega$-factors at typical conditions (30 days irradiation and 1 day cooling) are presented in Fig. 1.

According to the model described, the contact residual dose rates are calculated in 


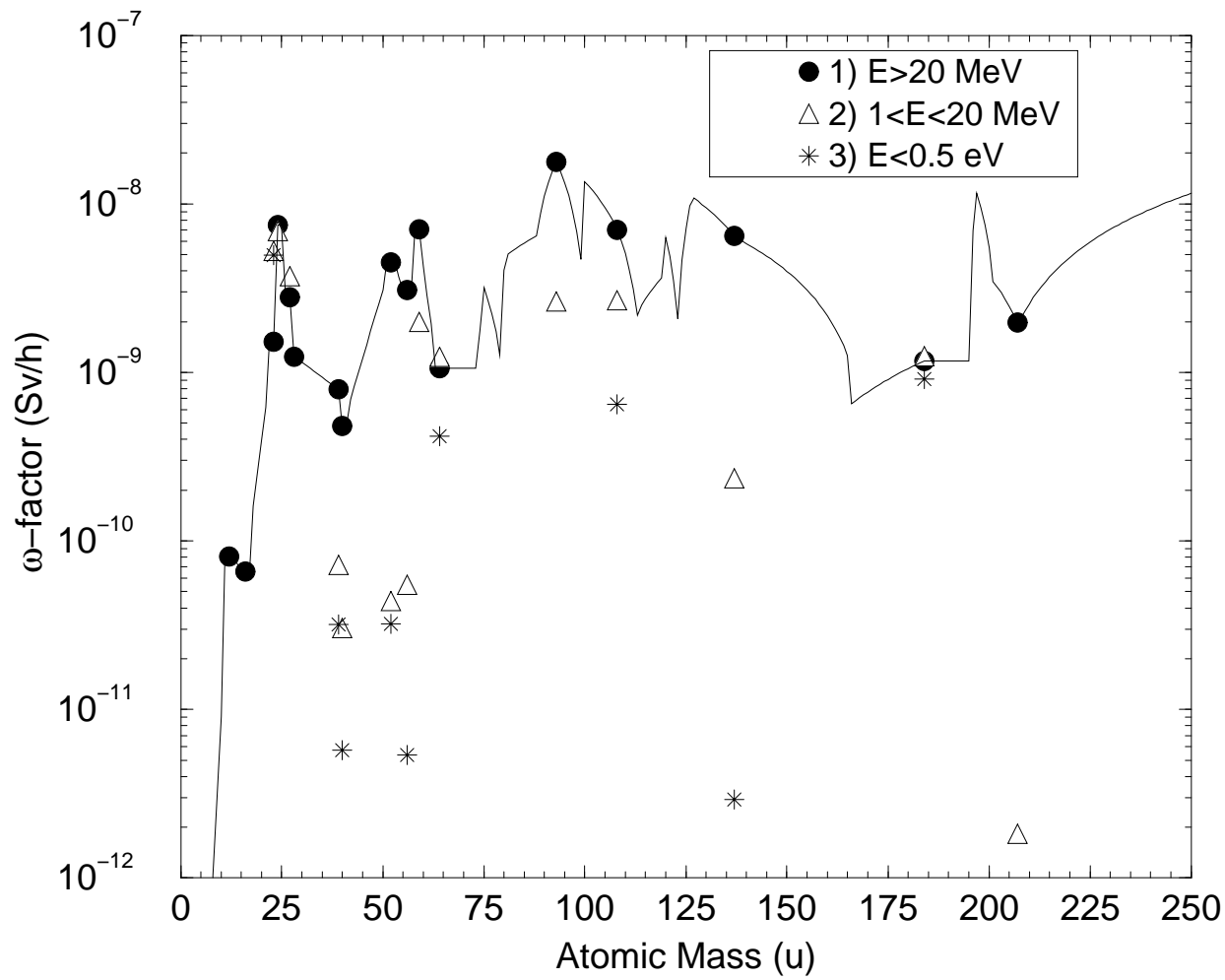

Figure 1: Example of $\omega$-factor dependence on mass of a target nucleus for three energy groups and $T_{i}=30$ days and $T_{c}=1$ day. Normalization is per $s t a r / \mathrm{cm}^{3} / \mathrm{s}$ for $E>20 \mathrm{MeV}$, and per neutron $/ \mathrm{cm}^{2} / \mathrm{s}$ for the other groups. The symbols represent the FLUKA results of a previous study [8] and the curve is an interpolation of the results of the study and those of an earlier one [11] for the high energy group.

MARS on the surface of irradiated objects with linear dimensions larger than some fraction of $\lambda_{i n}$, where $\lambda_{i n}$ is the nuclear interaction length. Such a procedure, being an approximation, has the advantage of using geometry- and dimension-independent contact residual dose rates.

\section{Scaling procedure for thin objects}

When considering thin or small objects, one must take into account geometry factors to perform scaling of calculated contact dose rates from thick objects to realistic ones. We introduce the following two-step procedure to calculate the geometry scaling factors by means of the MCNP [13] code. First, the dose rate, $D_{1}$, is calculated on the surface of a thick object of a given material at a given specific activity. Second, the dose rate, $D_{2}$, is calculated on the surface of a given realistic thin object of the same material and at the same specific activity. The geometry scaling factor for the given thin object is then defined as the ratio of the contact dose rate calculated at the second step to that calculated at the first step, $R_{G}=D_{2} / D_{1}$. Isotropic and monoenergetic 1-MeV gammas-as representatives 
of nuclear gamma decays due to spallation reactions-are used in these calculations to simulate a residual activity source term. Its spatial distribution is assumed to be uniform.

A qualitative justification for the energy of $1 \mathrm{MeV}$ is as follows. De-excitation of a residual nucleus formed in an interaction of a high-energy projecile with a target nucleus usually proceeds as the sequence of three stages: cascade, pre-equilibrium, and equilibrium. It is the slow-equilibrium-stage that is responsible for residual activation. Until the nuclear excitation exceeds the nucleon binding energy $(\simeq 8 \mathrm{MeV})$ the de-excitation proceeds via nucleon or fragment emission as the most preferable options from the standpoint of minimizing the nuclear potential energy. After the excitation drops below the nucleon binding energy, $\beta^{ \pm}$-decay as well as $\gamma$-decay due to transitions between low-lying nuclear levels come into play. The observed energy range of the emitted $\gamma$-quanta is between $10 \mathrm{keV}$ and $10 \mathrm{MeV}$ [14], in which case $2 \pi R / \lambda \ll 1$, where $R$ is nuclear radius and $\lambda$ is the wavelength of the $\gamma$-quanta. Probability of emission of $\gamma$-quanta with the angular momentum $L$ (in the units of $\hbar$ ) behaves approximately as $(2 \pi R / \lambda)^{2 L}$, so that multipolar transitions with high angular momenta are strongly suppressed. Therefore, the $\gamma$-decays usually proceed as a sequence of transitions between the nearest nuclear levels with minimal difference in nuclear spin. Difference in energy between such levels is of the order of $1 \mathrm{MeV}$. The spectra of $\gamma$-quanta emitted from several unstable nuclei are shown in Fig. 2.

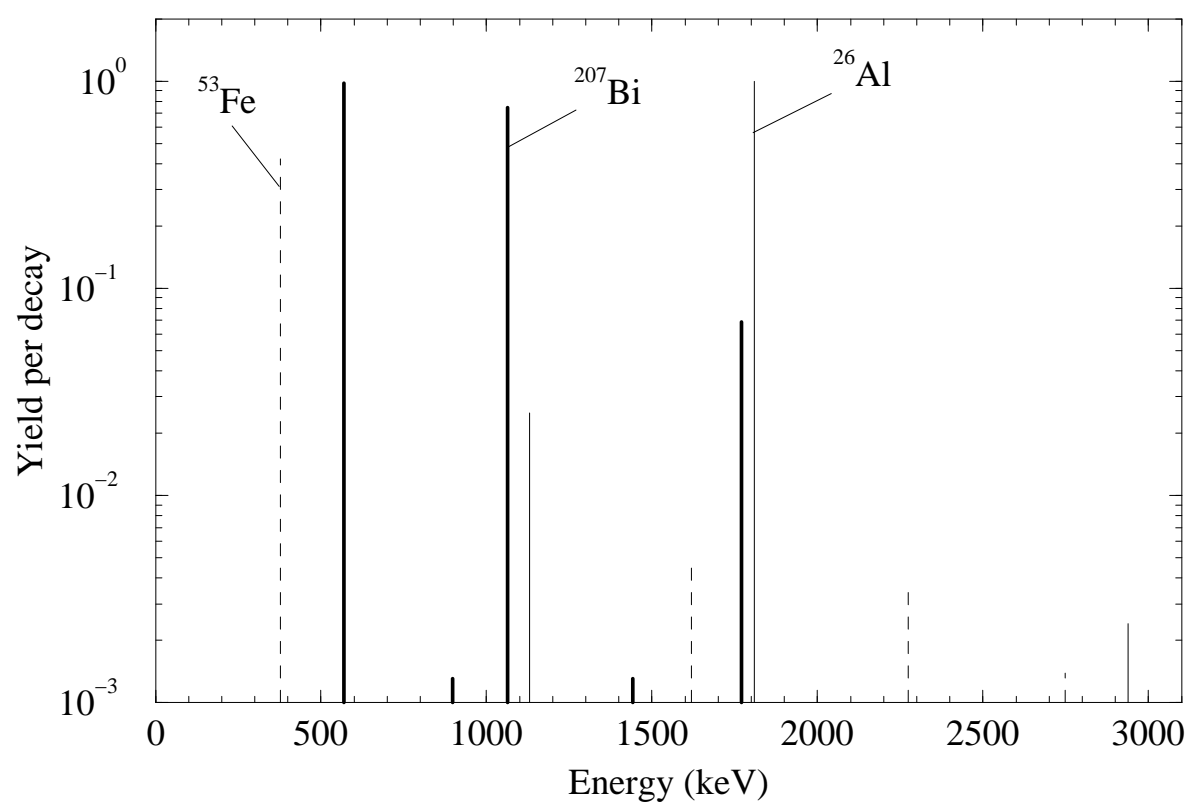

Figure 2: Energy spectra of $\gamma$-quanta emitted from several radioactive nuclei [15].

The described scaling procedure has been used to calculate the factors for slabs and cylinders, both solid and hollow, that can serve as models for collimators, magnets and beam pipes. Various materials have been taken into consideration. The results of the calculations along with results of fitting are presented below. A special consideration is required for small objects that are not considered to be infinite in either dimension.

In the following we use unitless variables. Therefore, thickness of each object is normalized to a mean free path of $1-\mathrm{MeV} \gamma$-rays in the material, $\lambda_{t}$, defined as $\lambda_{t}=\left(N \sigma_{t}\right)^{-1}$, 
Table 1: Mean free path, $\lambda_{t}$, of 1-MeV $\gamma$-rays in various materials [16]

\begin{tabular}{lccccccccccc}
\hline Material & $\mathrm{Be}$ & $\mathrm{C}$ & $\mathrm{Al}$ & $\mathrm{Ti}$ & $\mathrm{Fe}$ & $\mathrm{Cu}$ & $\mathrm{Zr}$ & $\mathrm{Mo}$ & $\mathrm{Sn}$ & $\mathrm{W}$ & $\mathrm{Pb}$ \\
\hline$\lambda_{t}(\mathrm{~cm})$ & 9.6 & 8.7 & 6.0 & 3.7 & 2.1 & 1.9 & 2.7 & 1.7 & 2.4 & 0.78 & 1.2 \\
\hline
\end{tabular}

where $N$ is atomic density and $\sigma_{t}$ is total microscopic interaction cross section. For the sake of convenience, in Table 1 the data on $\lambda_{t}$ for various materials are given.

\subsection{Slab}

A slab is usually shaped as a box having one dimension (say $z$ ) — thickness-less than the other two ( $x$ and $y$ in this particular case). In our simulations the thickness, $z$, is varied from $0.01 \lambda_{t}$ up to $4 \lambda_{t}$ while each of the other dimensions was equal to $100 \lambda_{t}$. The calculated scaling factors for various slabs are given in Fig. 3.

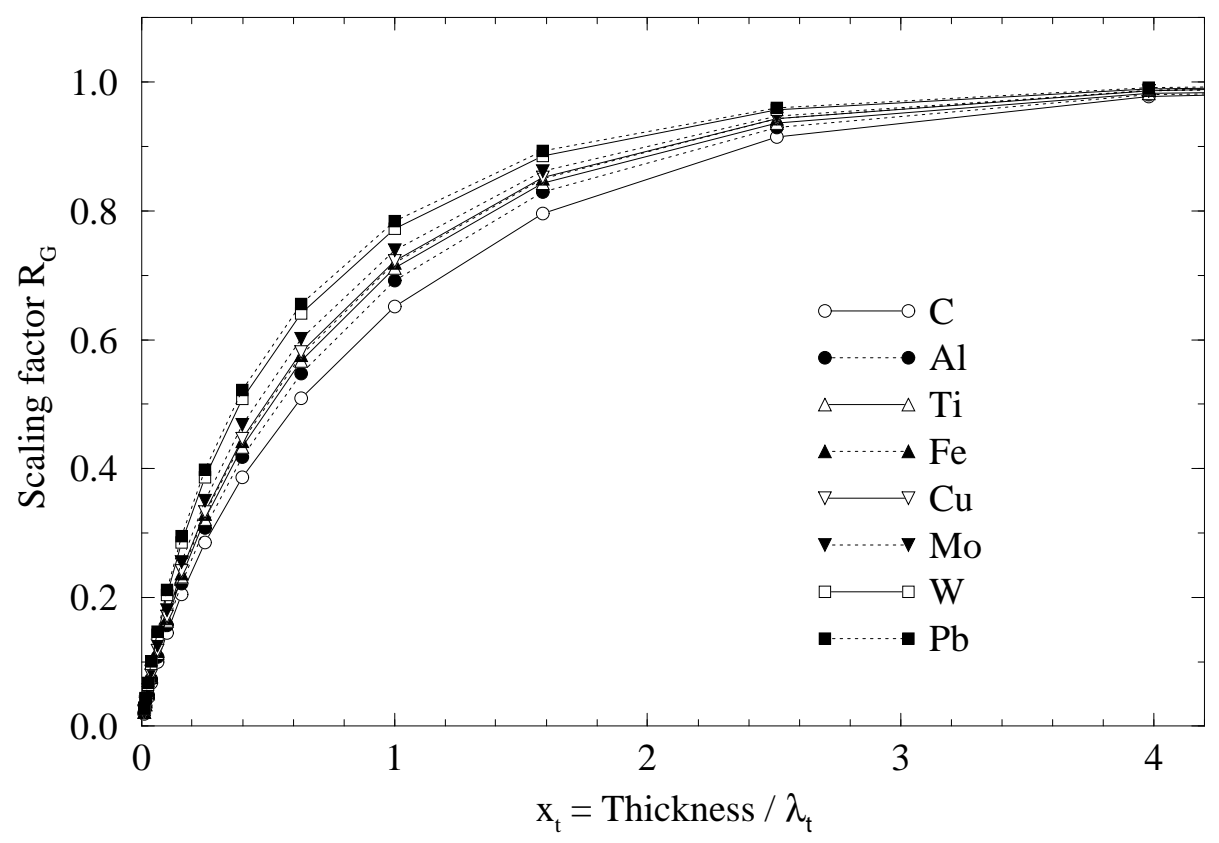

Figure 3: The calculated dose scaling factors, $R_{G}$, for slabs of various materials $v s$. the normalized thickness. The lines are drawn to guide the eye.

A fitting was performed to describe in a suitable form the dependence of the scaling factors on thickness and material. The following expression was found to be adequate to fit the data:

$$
R_{G}=\left(1-\exp ^{-B x_{t}}\right)^{C}
$$

where $R_{G}$ is the scaling factor, $x_{t}=$ thickness $/ \lambda_{t}, B$ and $C$ are fitting parameters given in Fig. 4. The advantage of using this expression is in the fact that it provides correct 


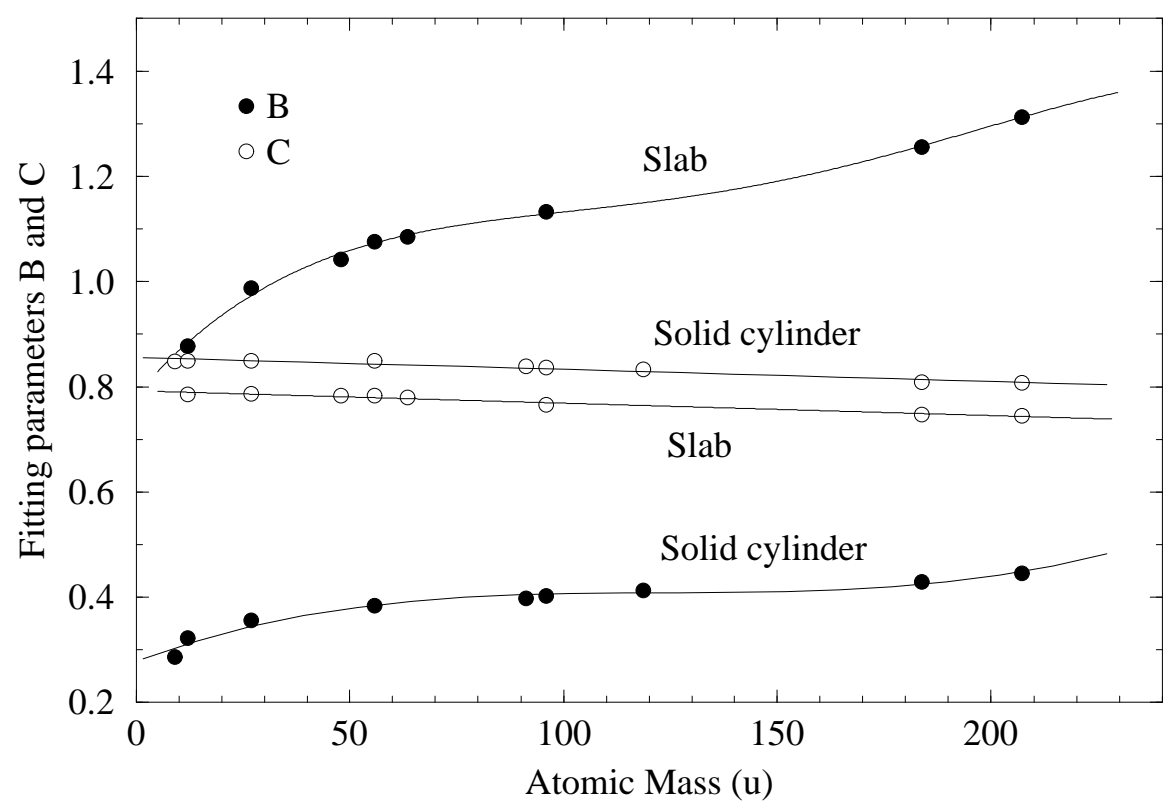

Figure 4: Fitting parameters $B$ and $C$ from the expression (2) $v s$. atomic mass. The circles represent results of calculations performed for corresponding nuclides and the curves is result of subsequent fitting.

asymptotic values for the dose scaling factor, $R_{G}$, at both $x_{t} \rightarrow 0$ and $x_{t} \rightarrow \infty$ independently of the values of the parameters $B$ and $C$. The dependence shown in Fig. 4 can, in turn, be described by the following expressions:

$$
\begin{aligned}
& B=B_{0}+\sum_{n=1}^{4} B_{n} A^{n}, \\
& C=C_{0}+C_{1} A,
\end{aligned}
$$

where $A$ is atomic mass and the expansion coefficients $B_{n}$ and $C_{n}$ are given in Table 2.

Table 2: The expansion coefficients $B_{n}$ and $C_{n}$

\begin{tabular}{ccccc}
\hline & \multicolumn{2}{c}{ Slab } & \multicolumn{2}{c}{ Solid cylinder } \\
\cline { 2 - 5 }$n$ & $B_{n}$ & $C_{n}$ & $B_{n}$ & $C_{n}$ \\
\hline 0 & $7.8426 \times 10^{-1}$ & $7.9241 \times 10^{-1}$ & $2.7839 \times 10^{-1}$ & $8.5604 \times 10^{-1}$ \\
1 & $9.39 \times 10^{-3}$ & $-2.35408 \times 10^{-4}$ & $3.06 \times 10^{-3}$ & $-2.29036 \times 10^{-4}$ \\
2 & $-1.00765 \times 10^{-4}$ & & $-2.42745 \times 10^{-5}$ & \\
3 & $5.00304 \times 10^{-7}$ & & $6.50166 \times 10^{-8}$ & \\
4 & $-8.36463 \times 10^{-10}$ & & & \\
\hline
\end{tabular}




\subsection{Solid cylinder}

The calculated scaling factors, $R_{G}$, for solid cylinders are shown in Fig. 5. The length of the cylinders was equal to $100 \lambda_{t}$, so that in all the cases the cylinders were considered to be infinitely long. The list of studied materials has been changed to provide more generality to these considerations and take into account the materials important from practical standpoint. For example, beam pipes used in the vicinity of interaction regions at high-energy colliders are usually made of beryllium. One can see that, other things being equal, for the cylinders the increase in the scaling factor with diameter (thickness) is significantly slower than that for slabs. Such a behaviour can be understood taking into account that the cylinders can be considered to be infinite in length only while for slabs it applies in two dimensions. To describe the scaling factors, $R_{G}$, for solid cylinders the same expression (2) is used and in this case $x_{t}$ is diameter divided by $\lambda_{t}$. The corresponding fitting parameters $B$ and $C$ are presented in Fig. 4 and Table 2.

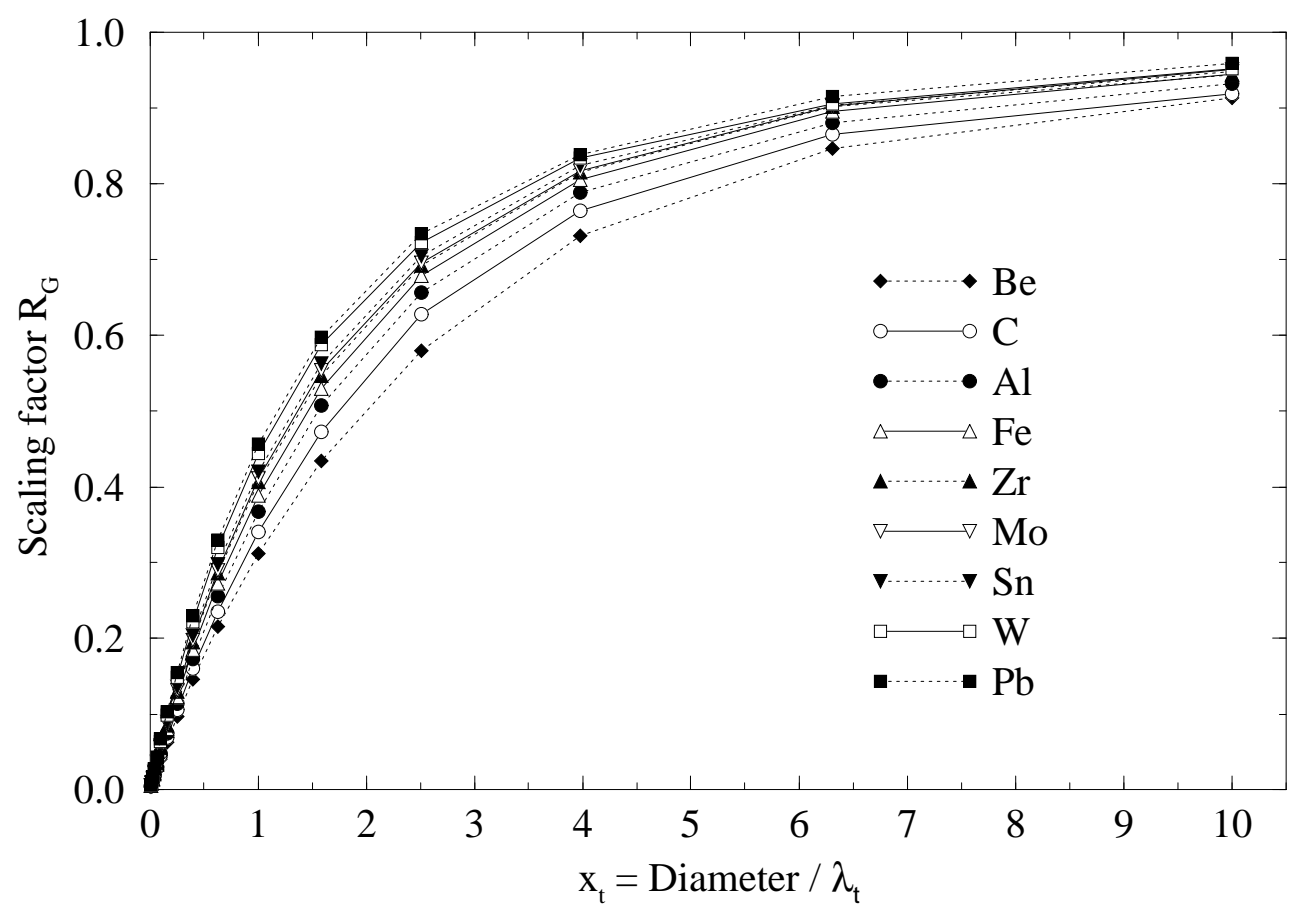

Figure 5: The calculated dose scaling factors, $R_{G}$, for solid cylinders of various materials $v s$. the normalized diameter. The lines are drawn to guide the eye.

\subsection{Hollow cylinder}

A hollow cylinder is of interest from practical standpoint as a perfect model for accelerator beam pipes and simple model of collimators and magnets. In our calculations, just like for solid cylinders, the length of the hollow ones is assumed to be infinite. In this case, however, there are two variables for initial fitting —inner and outer radii-instead of a single 
one (thickness or diameter). Therefore, we simplify the problem by means of taking into consideration only three materials: beryllium, aluminium, and iron. A justification is as follows. The highest relative difference in the calculated scaling factors between beryllium and lead is about 50\% at $x_{t}=1.6$ (see Fig. 5). Having calculated the factors for these three materials, for other materials one can perform an interpolation or extrapolation that provides a relative inaccuracy of $10-15 \%$ at the most. From practical point of view it is a quite acceptable level.

The calculated scaling factors for hollow cylinders with various inner and outer radii are shown in Fig. 6. One can see that the difference in the factors between beryllium and iron is about $15-20 \%$ as was anticipated, so that the above-mentioned interpolation and extrapolation procedures for other materials are justified. A fitting was performed for the data using the same expression (2), with $x_{t}$ being $\left(R_{\text {out }}-R_{\text {in }}\right) / \lambda_{t}$. The expression provides, just like previously with slabs and solid cylinders, correct asymptotic values for the dose scaling factor, $R_{G}$, at both $x_{t} \rightarrow 0$ and $x_{t} \rightarrow \infty$ independently of the values of the parameters $B$ and $C$. The calculated fitting parameters $B$ and $C$ are shown in Fig. 7. The dependencies shown in the Figure can, in turn, be described by the following expressions:

$$
\begin{aligned}
& B=B_{0}+B_{1} \exp ^{-x_{t} / x_{0}}, \\
& C=C_{0}+\sum_{n=1}^{3} C_{n} x_{t}^{n},
\end{aligned}
$$

where $x_{t}$ is $\left(R_{\text {out }}-R_{\text {in }}\right) / \lambda_{t}$ and the coefficients $B_{n}, C_{n}$, and $x_{0}$ are given in Tables 3 and 4 .

Table 3: The coefficients $B_{n}$ and $x_{0}$ for hollow cylinders

\begin{tabular}{cccc}
\hline & Beryllium & Aluminium & Iron \\
\hline$B_{0}$ & 1.4428 & 1.9489 & 2.1151 \\
$B_{1}$ & -0.85396 & -1.21355 & -1.3309 \\
$x_{0}$ & 1.7464 & 2.1108 & 2.0774 \\
\hline
\end{tabular}

Table 4: The expansion coefficients $C_{n}$ for hollow cylinders

\begin{tabular}{cccc}
\hline$n$ & Beryllium & Aluminium & Iron \\
\hline 0 & $8.6847 \times 10^{-1}$ & $8.5445 \times 10^{-1}$ & $8.5947 \times 10^{-1}$ \\
1 & $1.4330 \times 10^{-2}$ & $3.2520 \times 10^{-2}$ & $3.0440 \times 10^{-2}$ \\
2 & $-3.010 \times 10^{-3}$ & $-5.930 \times 10^{-3}$ & $-5.590 \times 10^{-3}$ \\
3 & $1.3174 \times 10^{-4}$ & $3.0188 \times 10^{-4}$ & $2.8175 \times 10^{-4}$ \\
\hline
\end{tabular}




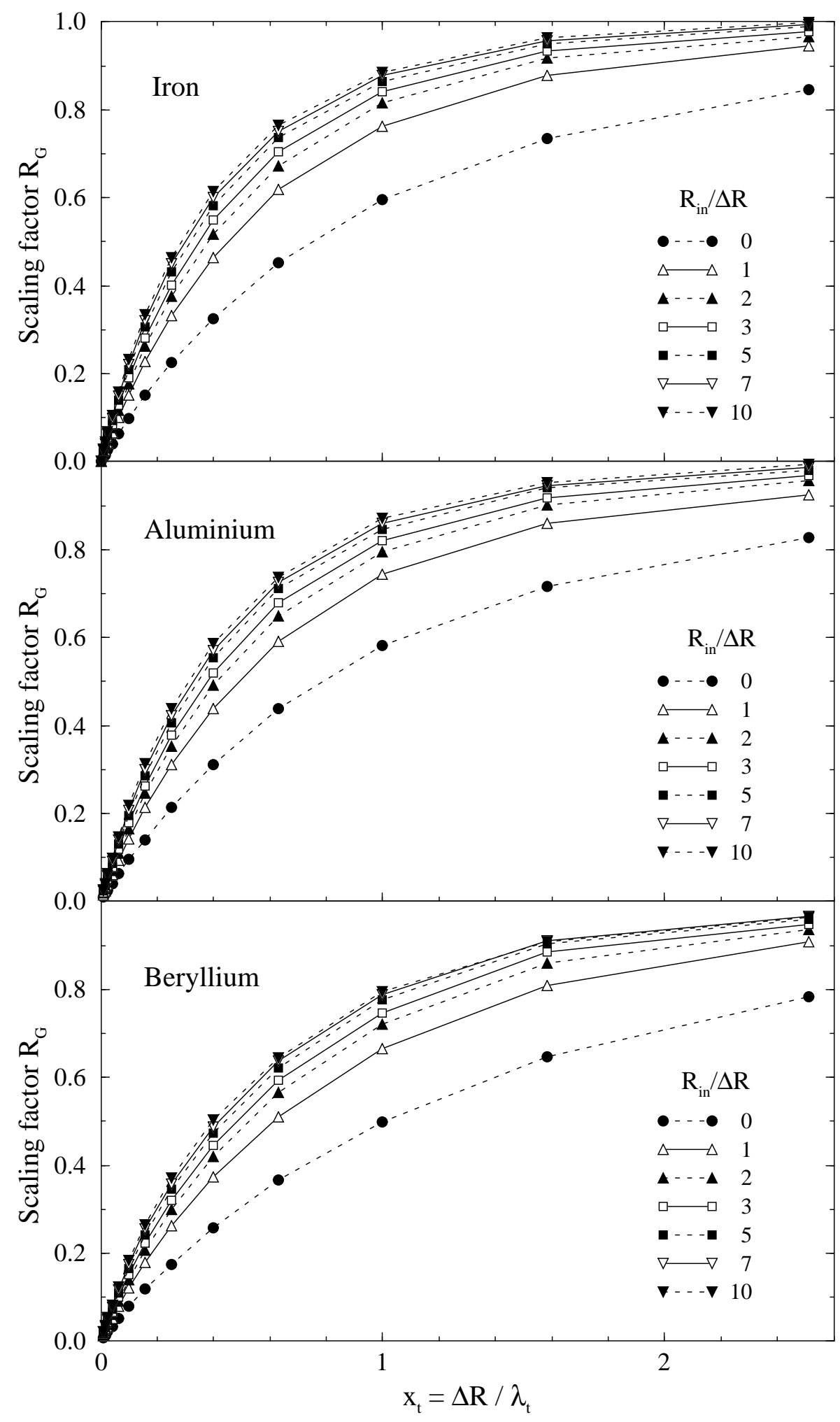

Figure 6: The calculated dose scaling factors, $R_{G}$, for hollow cylinders of several materials $v s$. the normalized wall thickness at various ratios of $R_{\text {in }} / \Delta R$, where $\Delta R$ is $R_{\text {out }}-R_{\text {in }}$ and $R_{\text {in }}$ and $R_{\text {out }}$ are inner and outer radii of the cylinder, respectively. The lines are drawn to guide the eye. 


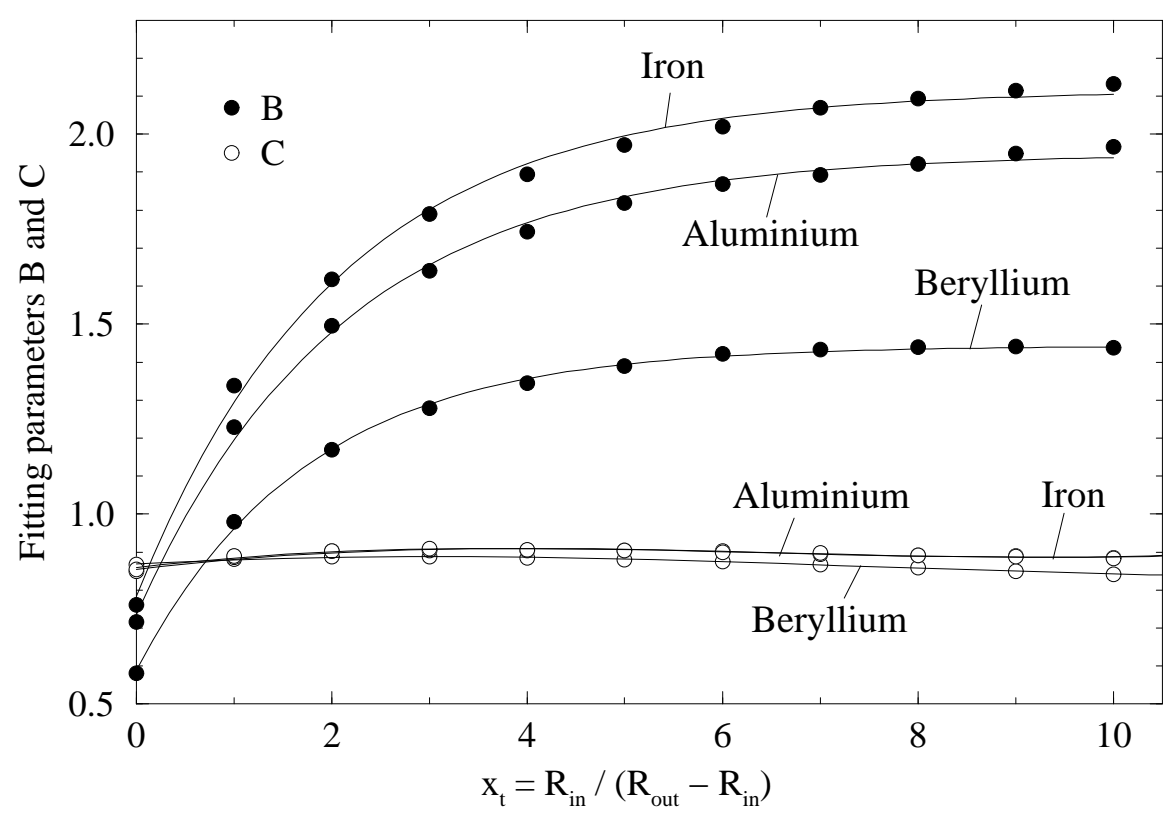

Figure 7: Fitting parameters $B$ and $C$ from the expression (2) for hollow cylinders at various inner and outer radii $R_{i n}$ and $R_{\text {out }}$, respectively. The circles represent results of calculations performed for corresponding materials and the curves is result of subsequent fitting.

\section{Residual dose attenuation in the air}

Another important issue we address in this study is the residual dose attenuation in the air surrounding a cylindrical object. An analytical description of the attenuation would be very convenient for an express dose estimate around the object. Therefore, we performed calculations for a number of materials and fitted the resulting distributions as follows. The source term in these calculations was the same as in the previous section-isotropic and monoenergetic $1-\mathrm{MeV} \gamma$-rays with a uniform spatial distribution over the volume under consideration.

Let the dose at the distance $r$ from the side surface of a cylinder, $D(r)$, be described by the following expression:

$$
D(r)=D_{0} f(r),
$$

where $D_{0}$ is the surface contact dose calculated according to the recipes of the previous section and $f(r)$ is an attenuation function. Samples of calculated dose attenuation in the air around solid aluminium cylinders are shown in Fig. 8.

Initially we used the following expression to fit the attenuation function:

$$
f(r)=\frac{1}{\left(1+B_{a} r\right)^{C_{a}}},
$$

where $B_{a}$ and $C_{a}$ are fitting parameters. The subscript $a$ was introduced to distinguish between current parameters used to describe spatial dose attenuation in the air and the 


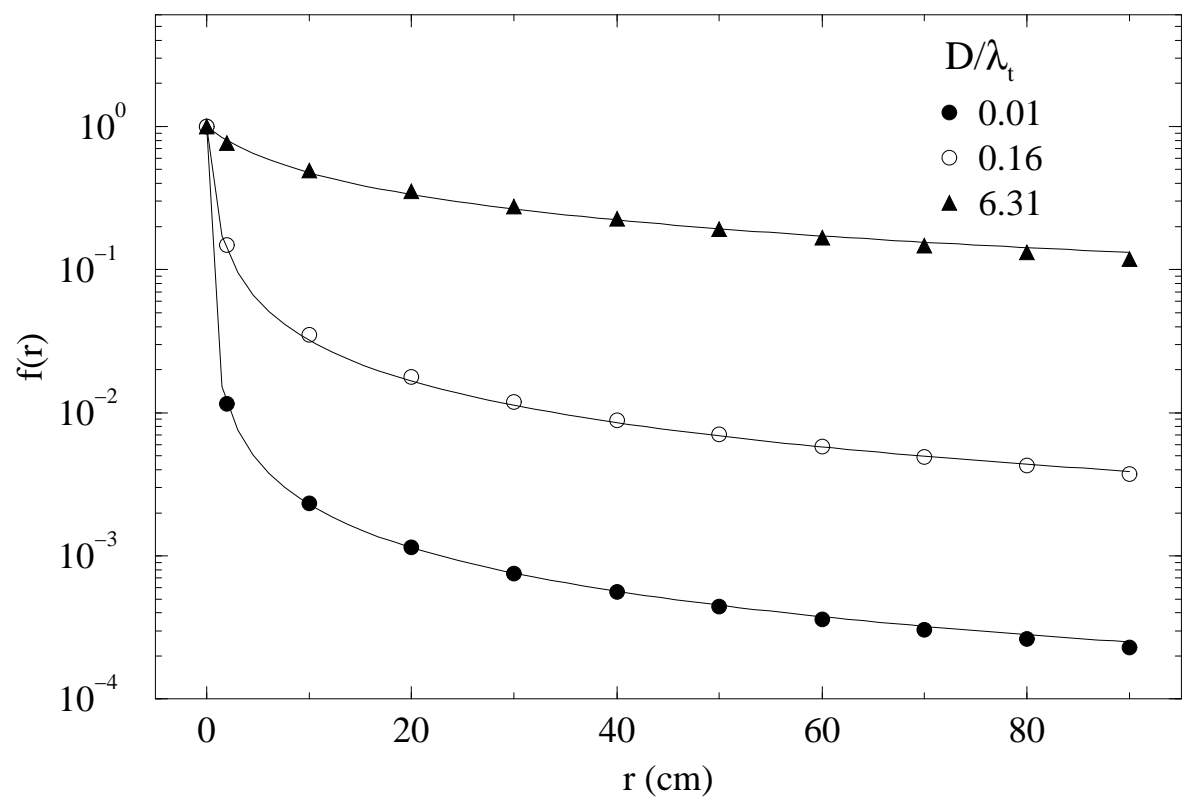

Figure 8: Calculated (symbols) dose attenuation function from the expression (7), $f(r)$, in the air surrounding aluminium solid cylinders of various diameters, $D$, vs the radial distance from the side surface of the cylinder. The values of the ratio $D / \lambda_{t}$ for the cylinders are shown on the graph. The curves represent a result of fitting the data by means of the expression (8).

parameters $B$ and $C$ used in previous sections. The parameters $B_{a}$ and $C_{a}$ are assumed to depend on material and diameter of the cylinder. Further calculations revealed that the parameter $B_{a}$ scales with the material density, so that finally we came to the following expression for the attenuation function:

$$
f(r)=\frac{1}{\left[1+0.2286 \rho r\left(D / \lambda_{t}\right)^{-0.9157}\right]^{C_{a}}},
$$

where $\rho$ is material density in $\mathrm{g} / \mathrm{cm}^{3}$ and $D$ is diameter of the cylinder. Thus, the problem is reduced to the fitting parameter $C_{a}$. The dependence of the parameter on material will be addressed in another study.

\section{Comparison to experimental data}

To verify the technique developed in this paper, we compared calculated and measured residual activity (see Ref. [6]). The measurements were performed at the Fermi National Accelerator Laboratory with a $120 \mathrm{GeV}$ proton beam to study induced radioactivation of materials used for beam line components and shielding (see Fig. 9).

Residual activation exposure rates were measured for five small cylindrical and rectangular samples of iron, steel, aluminum, and concrete. The samples were placed both 


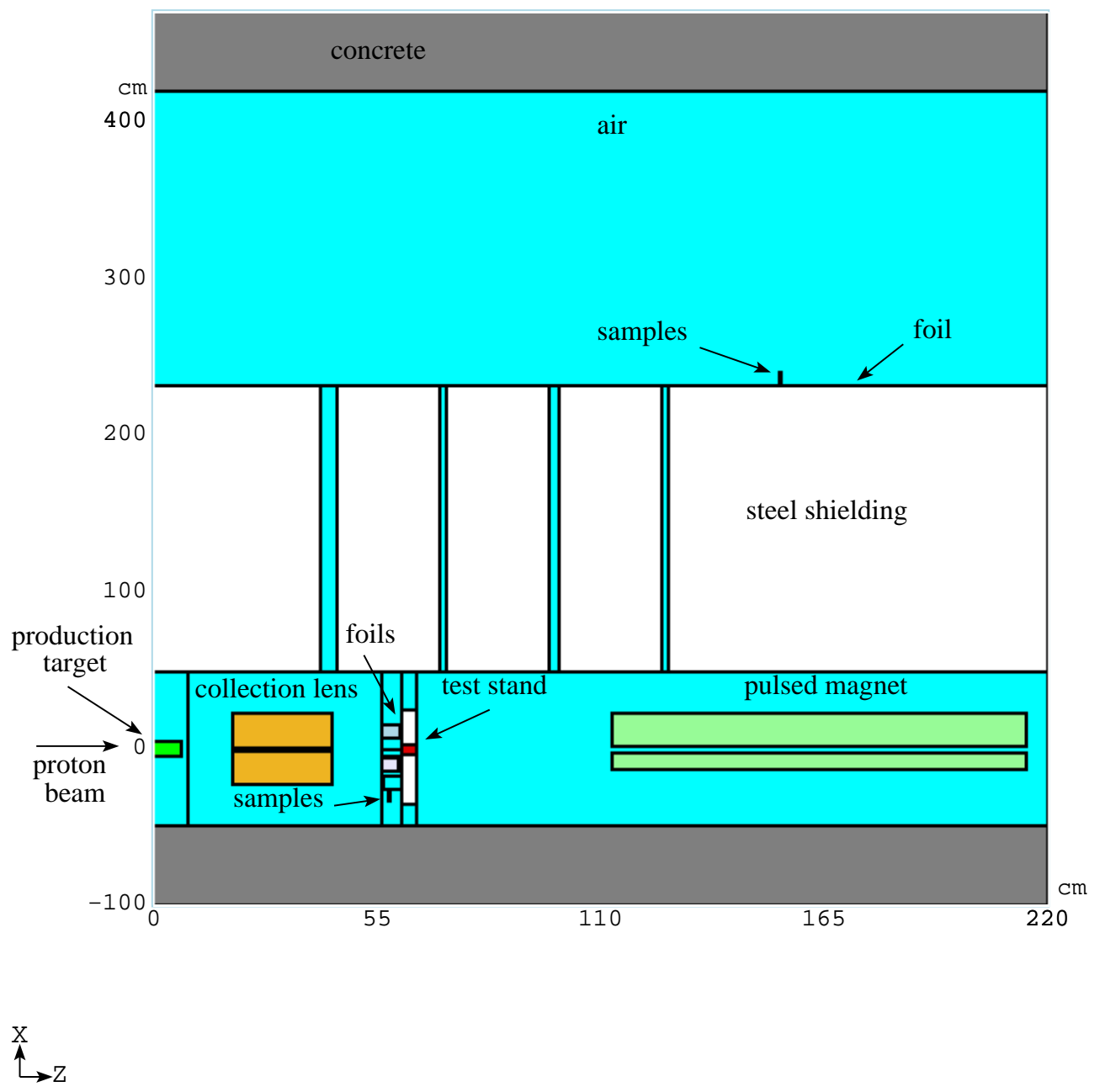

Figure 9: An elevation view of the experimental area as modeled in MARS.

within the vault area (just downstream of the antiproton production target) and at a location outside the thick steel shielding at AP0 enclosure. The samples within the vault were irradiated for a total of 38 hours by the radiation arising from the bombardment of the target by about $1.3 \times 10^{17} 120 \mathrm{GeV}$ protons from the Main Injector, and then removed to a low background area for counting; those outside of the shielded vault area were irradiated on and off for about four months with a total of approximately $3.6 \times 10^{18}$ protons incident on the in-vault target. Background corrected exposure rates of the samples were determined by use of both Geiger-Müller (GM) and NaI scintillator based survey instruments.

A comparison between measured and calculated residual dose rates for two of the samples is shown in Fig. 10. One can see that the agreement is very good even for a cooling time of about a month. 

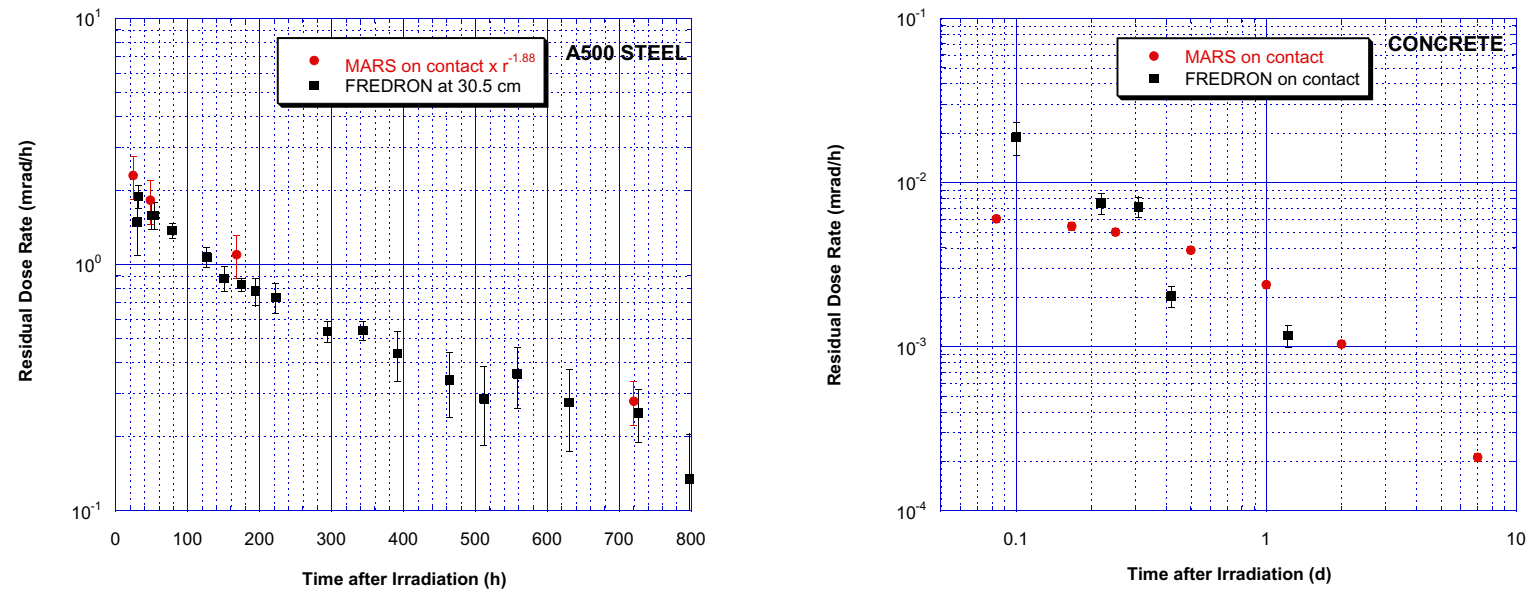

Figure 10: Measured (FREDRON) and calculated (MARS) residual dose rates for the A500 steel sample in the vault (left) and concrete sample outside the shielding (right).

\section{Future development}

In the following we will investigate the dependence of the fitting parameter $C_{a}$ (see expression (9)) on material to describe dose attenuation in the air around solid cylinders as well as apply the approach described in Sec. 4 to hollow cylinders.

We also plan to implement the method developed in this paper into the MARS code to allow direct calculation of residual dose in and around arbitrary objects.

\section{References}

[1] a) W.B. Wilson, Los Alamos National Laboratory, NM, LA-UR-93-3080, 1993; b) W.B. Wilson, T.R. England, K.A. Van Riper, Proc. IV SARE Workshop, Knoxville, TN, USA, September 1998, Ed. T.A. Gabriel, p.69 (1998).

[2] A.G. Croff, Nucl. Technol. 62 (1983) 335.

[3] P.A. Aarnio, CERN CMS Note-1998/086, 1998.

[4] J. Ranft and K. Goebel, CERN HP-70-92, Internal Note, 1970.

[5] G. Stevenson, Private communication, CERN.

[6] a) I. Rakhno, N. Mokhov, A. Elwyn, N. Grossman, M. Huhtinen, L. Nicolas, Fermilab-Conf-01/304-E, 2001; b) Proc. Amer. Nucl. Soc. Topical Meeting AccApp/ADTTA'01, Reno, Nevada, USA, Nov. 11-15, 2001; Omnipress, 2002, Omnipro-CD, ISBN:0-89448-666-7.

[7] M. Huhtinen and L. Nicolas, CERN CMS Note-2002/019, 2002. 
[8] M. Huhtinen, Proc. 42nd Workshop on Innovative Detectors for Supercolliders, Erice, Sicily, Italy, 28 Sep. - 4 Oct., 2003.

[9] N.V. Mokhov, Fermilab-FN-628 (1995); N.V. Mokhov, K.K. Gudima, C.C. James et al., Radiation Protection and Dosimetry, vol. 116, part 2, pp. 99-103 (2005) and Fermilab-Conf-04/053 (2004); N.V. Mokhov, K.K. Gudima, S.G. Mashnik et al., Proc. Int. Conf. on Nuclear Data for Science and Technology, Santa Fe, NM, 2004, AIP Conf. Proc. 769, part 2, pp. 1618-1623 and Fermilab-Conf-04/269-AD (2004); http://www-ap.fnal.gov/MARS/.

[10] http://www.fluka.org/.

[11] M. Huhtinen, CERN/TIS-RP/IR/98-28, 1998.

[12] M. Huhtinen, CERN CMS Note-1998/044, 1998.

[13] J.F. Briesmeister, editor, Los Alamos National Laboratory, NM, LA-13709-M, 2000.

[14] M. Barbier, Induced Radioactivity, John Wiley \& Sons, Inc., New York, 1969.

[15] http://www.nndc.bnl.gov/mird/

[16] http://physics.nist.gov/PhysRefData/contents.html 